\title{
THE COMPACTUM AND FINITE DIMENSIONALITY IN BANACH ALGEBRAS
}

\author{
ABDULLAH H. AL-MOAJIL \\ Gulf Organization for Industrial Consulting \\ P.0. Box 5114 \\ Doha, Qatar
}

(Received March 2, 1981 and in revised form September 23, 1981)

ABSTRACT. Given a Banach algebra A, the compactum of $A$ is defined to be the set of elements $x \in A$ such that the operator $a \rightarrow$ xax is compact. General properties of the compactum and its relation to the socle of A are discussed. Characterizations of finite dimensionality of a semi-simple Banach algebra are given in terms of the compactum and the socle of $A$.

KEY WORDS AND PHRASES. Compact operators, socle, minimal idempotent, spectrum. 1980 MATHEMATICS SUBJECT CLASSIFICATION CODES. Primary. 46H05, 46H10; Secondary $47 B 05$.

\section{INTRODUCTION.}

Familiarity with Banach algebras is assumed. An elementary survey of this area is Rickart [5], which is accessible to anyone with a basic knowledge of measure theory.

Let $\mathrm{A}$ be a Banach algebra. For $\mathrm{x} \varepsilon \mathrm{A}$ let $\mathrm{T}_{\mathbf{x}}$ be the operator on $\mathrm{A}$ defined by $T_{x}(a)=x a x$. Define the compactum of $A$ to be the set $C(A)=\left\{x \in A: T_{x}\right.$ is a compact operator]. In [1], J.C. Alexander investigated the properties of a Banach algebra $A$ which satisfies $A=C(A)$. He called this type of algebra a compact Banach algebra. The concept was also considered by Erdos, Giotopoulos and Lambrou [2]. It has its origin in a result by Vala which states that if $\mathrm{X}$ is a Banach space and $T$ and $T^{\prime}$ are non-zero elements of $B(X)$, then the operator $S^{-}$TST' $^{\prime}$ is compact on $B(X)$ if and only if both $T$ and $T^{\prime}$ are compact on $X$ [3]. 
The purpose of this paper is to look at some general properties of $\mathrm{C}(\mathrm{A})$ and to give a characterization of finite dimensionality for a semi-simple Banach algebra A, using $\mathrm{C}(\mathrm{A})$. The latter result generalizes a theorem of A.W. Tullo [4].

\section{GENERAL PROPERTIES.}

If $A$ is a Banach algebra with minimal left and right ideals, and if the sume of the minimal left ideals coincides with the sum of the minimal right ideals, then the resulting ideal is called the socle of $A$. We let $S(A)$ denote the socle of $A$. If $\mathrm{X}$ is a Banach space then $\mathrm{B}(\mathrm{X})$ will stand for the algebra of bounded operators on $X$ and $K(X)$ will denote the subalgebra of $B(X)$ consisting of compact operators. If $A$ is an algebra and $\mathrm{x} \varepsilon \mathrm{A}$, then $\sigma(\mathrm{X})$ will denote the spectrum of $\mathrm{x}$ in $\mathrm{A}$.

Our terminology is consistent with that of [5], and all algebras considered are over the field of complex numbers $\mathrm{C}$. We recall again that a Banach algebra $\mathrm{A}$ is compact if $A=C(A)$.

PROPOSITION 1. Let A be a Banach algebra. Then,

a) $\mathrm{C}(\mathrm{A})$ is a closed set.

b) If $x \in C(A)$ then the ideals $x A$ and $A x$ are both contained in $C(A)$.

c) If $B$ is a closed subalgebra of $A$ such that $B \subset C(A)$, then $B$ is a compact Banach algebra.

PROOF. Let $\left(X_{n}\right)$ be a sequence in $C(A)$ which converges to $x$. Then for each a $\varepsilon$ A we have $\left\|\left(T_{x}-T_{x_{n}}\right)(a)\right\|=\left\|\operatorname{xax}-x_{n} a x_{n}\right\| \leq\left\|\operatorname{xax}-x_{n} a x\right\|+\left\|x_{n} a x-x_{n} a x_{n}\right\| \leq$ $\|a\|\left(\|x\|+\left\|x_{n}\right\|\right)\left\|x-x_{n}\right\|$. Hence $\left|T_{x}-T_{x_{n}}\right| \leq\left(\|x\|+\left\|x_{n}\right\|\right)\left\|x-x_{n}\right\|$ where $|\cdot|$ denotes the operator norm. It follows that $T_{x_{n}}$ converges to $T_{x}$, and since $T_{x_{n}}$ is compact for all $\mathrm{n}$, we get $\mathrm{T}_{\mathrm{x}}$ is compact. Therefore, $\mathrm{x} \varepsilon \mathrm{C}(\mathrm{A})$. This proves (a). If $x \in C(A)$ and $y \in A$, then $T_{x y}$ is the composition of the maps $a \rightarrow y a, T_{x}$ and $a \rightarrow$ ay, and since $T_{x}$ is compact it follows that $T_{x y}$ is compact, i.e., $x y \varepsilon C(A)$. Similarly yx $\varepsilon C(A)$, which proves (b). Part (c) follows from the definition of compact Banach algebra and the fact that if $x \varepsilon B \subset C(A)$, then the restriction of $\mathrm{T}_{\mathbf{X}}$ to $\mathrm{B}$ is still compact.

PROPOSITION 2. (a) If $A$ is finite dimensional then $C(A)=A$. (b) If $X$ is 
a Banach space then $C(B(X))=K(X)$.

PROOF. Part (a) is obvious, while part (b) follows by Vala's theorem which states that $T, T^{\prime} \varepsilon K(X)$ if and only if the map $S \rightarrow T S T$ ' is compact on $B(X)$ [3] .

The next two lemmas appear in [1]. We state them here without proofs.

LEMMA 1. If $\mathrm{A}$ is a compact Banach algebra which is not a radical algebra, then A contains an idempotent e such that eAe is finite dimensional [1, 4.3].

LEMMA 2. Let $A$ be a semi-simple Banach algebra and $x \in A$. Then $S(A)$ exists and $x \in S(A)$ if and only if $T_{x}$ has finite rank (i.e., $x A x$ is finite dimensional).

PROPOSITION 3. Let A be a semi-simple Banach algebra. Then $C(A)$ is nonzero if and only if $S(A)$ is nonzero, and in this case $S(A) \subset C(A)$.

PROOF. Suppose that $\mathrm{C}(\mathrm{A})$ is nonzero. Choose $\mathrm{x} \in \mathrm{C}(\mathrm{A})$. Then the right ideal $\mathrm{xA}$ is contained in $\mathrm{C}(\mathrm{A})$, by Proposition $1(\mathrm{~b})$. Let $\mathrm{J}$ be the closure of $\mathrm{xA}$. Then $\mathrm{J} \subset \mathrm{C}(\mathrm{A})$ since $\mathrm{C}(\mathrm{A})$ is closed. Therefore, by Proposition $1(\mathrm{c})$, $\mathrm{J}$ is a compact Banach algebra, and hence, by Lemma 1, it contains an idempotent e such that eJe is finite dimensional. But since $\mathrm{eA} \subset \mathrm{J}$, we have $\mathrm{eAe}=\mathrm{e}(\mathrm{eA}) \mathrm{e} \subset \mathrm{eJe}$, and hence eAe is finite dimensional. It follows, by Lemma 2 , that $e \in S(A)$.

If $S(A)$ is nonzero then, applying Lemma 2 , we get $S(A) \subset C(A)$.

PROPOSITION 4. If $A$ is a Banach algebra and $x \in C(A)$, then 0 is the only accumulation point for $\sigma(x)$. Moreover, if $\lambda \varepsilon \sigma(x), \lambda \neq 0$ then there exists y $\varepsilon$ A commuting with $x$ such that $T_{x}(y)=\lambda^{2} y$.

PROOF. First note that if $A$ has no identity and $A_{1}$ is the Banach algebra obtained by adjoining an identity to $A$ in the usual manner, then the operator $\mathrm{T}_{\mathrm{x}}$ on $A_{1}$ is still compact. Moreover, $\sigma(x)$ as an element of $A_{1}$ or as an element of $A$ is the same. Therefore, we may assume that $\mathrm{A}$ has an identity.

Let $C$ be a maximal commutative subalgebra containing $x$ and let $T$ be the restriction of $T_{x}$ to $C$. If $\lambda \notin \sigma(T)$, then there exists $S \in B(C)$ such that $(\lambda-T) S=$ I, i.e., $(\lambda-T) S y=y$ for all y $\varepsilon$ C. This is equivalent to $\left(\lambda-x^{2}\right)$ Sy $=y$. If we choose y $\varepsilon C$ invertible, then $(S y) y^{-1}$ is an inverse for $\lambda-x^{2}$. That is $\lambda \notin \sigma\left(x^{2}\right)$. This says that $\sigma\left(x^{2}\right) \subset \sigma(T)$. Since $T$ is compact, the conclusion follows from the general theory for compact operators and the spectral mapping theorem. 


\section{CHARACTERIZATION OF FINITE DIMENSIONALITY.}

For the remainder of this paper we will give a characterization of finite dimensionality for semi-simple Banach algebras. Our result generalizes a theorem of A. W. Tullo which states that a semi-simple Banach algebra A which satisfies $A=S(A)$ is finite dimensional [4]. Before stating and proving our main theorem we need two lemmas.

LEMMA 3. Let $A$ be a semi-simple Banach algebra such that $x C(A)=(0)$ implies $x=0$. Suppose that $J \neq(0)$ is a closed right ideal in A. Then $J$ contains an idempotent e such that e $\varepsilon \mathrm{S}(\mathrm{A})$.

PROOF. Choose $x \in J$ and $y \in C(A)$ such that $x y \neq 0$. Then, by Proposition $1(b)$, $x y A \subset C(A)$. We also have $x y A \subset J$. Since $A$ is semi-simple, it follows that xyA is not a radical algebra. Hence, by Lemma 1, xyA contains an idempotent e such that exyAe is finite dimensional. But e $\varepsilon$ xyA implies that eA $\subset$ xyA, and hence eAe $=e(e A)$ e $\subset$ exyAe. Therefore, eAe is finite dimensional, and it follows from Lemma 2 that e $\varepsilon \mathrm{S}(\mathrm{A})$.

The next lemma appears in [6], and we include it here for the sake of completeness. We recall that an idempotent $\mathrm{e}$ in an algebra $\mathrm{A}$ is minimal if eAe is a division algebra. This is equivalent to saying that eA (Ae) is a minimal right (left) ideal [5]. By an Idempotent we always mean a non-zero one.

LEMMA 4. Let $A$ be a semi-simple normed algebra. If $e$ and $f$ are minimal idempotents in A then eAf is at most 1-dimensional.

PROOF. Suppose that eAf $\neq 0$ and choose $x$ such that $\operatorname{exf} \neq 0$. Then exfA $=e A$ by minimality of eA. Moreover, by the Gelfand-Mazur theorem, fAf $=\mathrm{fC}$ where $\mathrm{C}$ is the field of complex numbers. It follows that eAf $=$ exfAf $=$ exfC. That is eAf is 1-dimensiona1.

THEOREM. Let $A$ be a semi-simple Banach algebra such that $\mathrm{xC}(\mathrm{A})=(0)$ implies $\mathbf{x}=0$, then the following statements are equivalent,
a) A is finite dimensional.
b) (A. W. Tullo) $A=S(A)$.
c) $\mathrm{S}(\mathrm{A})=\mathrm{C}(\mathrm{A})$.
d) S(A) is closed. 
PROOF. If (a) holds then $\mathrm{T}_{x}$ has finite rank for every $x \in A$. Therefore, $A=S(A)$ by Lemma 2. If (b) holds then (c) follows from Proposition 3. The fact that $C(A)$ is closed shows that (c) implies (d). It remains to show that (d) implies (a).

Suppose that $S(A)$ is closed. We first show that A cannot contain an infinite set of pairwise orthogonal idempotents. Suppose that to the contrary such a set $\left\{e_{n}\right\}_{n=1}^{\infty}$ exists. Let $J_{i}=e_{i} A$. Then $J_{i}$ is a non-zero closed right ideal for each i. It follows by Lemma 3 , that each $J_{i}$ contains an idempotent $f_{i}$ such that $f_{i} \in S(A)$. Let $g_{i}=f_{i} e_{i}$. Then $g_{i} \varepsilon S(A)$, and since $f_{i}=e_{i} f_{i}$ it follows that $g_{i}$ is an idempotent and $\left\{g_{i}\right\}_{i=1}^{\infty}$ is a pairwise orthogonal family. Note that $g_{i} \neq 0$, otherwise $f_{i}=f_{i}^{2}=f_{i} e_{i} f_{i}=g_{i} f_{i}=0$. Now let $y_{n}=\sum_{i=1}^{n} \frac{g_{i}}{2^{i}\left\|_{g_{i}}\right\|}$. Then $y_{n} \varepsilon(A)$. Since $S(A)$ is closed it follows that $y=\lim _{n \rightarrow \infty} y_{n}=\sum_{i=1}^{\infty} \frac{g_{i}}{2^{i}\left\|_{g_{1}}\right\|} \varepsilon s(A)$. But we have $2^{i}\left\|g_{i}\right\| y \quad g_{i} y=g_{i}$. Therefore, the set $\left\{g_{i}\right\}$ is contained in yAy, and since $1 t$ is an infinite set and linearly independent by the orthogonality of its elements we have yAy is infinite dimensional. This contradicts the fact that $y \varepsilon S(A)$, by Lemma 2 . Hence, A contains at most a finite set of pairwise orthogonal idempotents.

Now let $\left\{e_{1}, \ldots, e_{n}\right\}$ be a set of pairwise orthogonal idempotents of maximal possible cardinality. Then each $e_{1}$ is minimal. Otherwise if $e_{1}$, say, is not minimal, then $e_{1} A$ is not a minimal ideal, hence it is not a minimal closed ideal [5; 2.1.10]. Thus $e_{1} A$ properly contains a closed right ideal I which, by Lemma 3 , contains an idempotent $f$, necessarily different from $e_{1}$ and $f=e_{1} f$. Then $\left\{e_{1}\right.$, $\left.e_{1}-f e_{1}, e_{2}, \ldots e_{n}\right\}$ is a pairwise orthogonal family of idempotents which contradicts the maximality of $n$.

Now let $f=e_{1}+\ldots+e_{n}$. We claim that $f$ is an identity for $A$. If $(1-f)$ $A \neq 0$, then since it is a closed ideal, Lemma 3 implies that it containa an idempotent $g=(1-f) g$. Let $h=g(1-f)$. Then $h$ is an idempotent which is orthogonal to $e_{i}$ for $i=1, \ldots, n$. Then by maximality of $n$, we get $h=0$. Hence $g=$ $\mathrm{g}^{2}=\mathrm{g}(1-\mathrm{f}) \mathrm{g}=\mathrm{hg}=0$ which is a contradiction. Hence $(1-\mathrm{f}) \mathrm{A}=0$. Noting that the conclusion of Lemma 3 holds for closed left ideals as well, a similar 
argument as the above gives $A(1-f)=0$. Therefore $f$ is an identity of $A$. It follows that $A=f A f=\left(\sum_{i=1}^{n} e_{i}\right) A\left(\sum_{i=1}^{n} e_{i}\right)=\sum_{i, j=1}^{n} e_{i} A e_{j}$. But, by Lemma 4 , $e_{i} \mathrm{Ae}_{j}$ is at most 1 -dimensional for $i, j=1, \ldots, n$. Hence, $A$ is finite dimensional. This shows that (d) implies (a) and concludes the proof of the theorem. ACKNOWLEDGEMENT.

The author would like to thank the International Center for Theoretical Physics (ICTP) in Trieste, Italy for their encouragement and hospitality during the work on the paper.

\section{REFERENCES}

1. ALEXANDER, J.C. Compact Banach Algebras, Proc. London Math. Soc. (3) $\underline{18}$, (1968), pp. 1-18.

2. ERDOS, J., GIOTOPOULOS, S., and LAMBROU, M. Rank one elements of Banach Algebras, Mathematika 24 (1977), pp. 178-181.

3. VALA, K. On compact sets of compact operators, Ann. Acad. Sci. Fenn.. Ser. AI, No. $\underline{351}$ (1964).

4. TULLO, A.W. Conditions on Banach algebras which imply finite dimensionality, Proc. Edinburgh Math. Soc. (2) 20 (1976), pp. 1-5.

5. RICKART, C.E. General theory of Banach algebras. Von Nostrand, Princeton, N.J., MR 22 No. 5903 (1960).

6. AL-MOAJIL, A.H. Finite dimensionality of a normed algebra which is equal to its socle, Mathematics Seminar Notes, Kobe Univ., Japan, Vol. 7 (1979), pp. 133-137. 


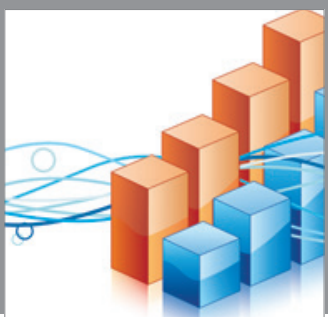

Advances in

Operations Research

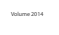

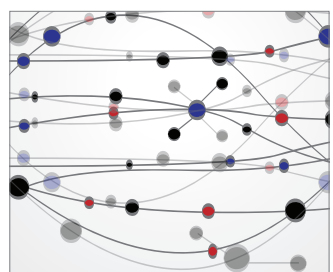

\section{The Scientific} World Journal
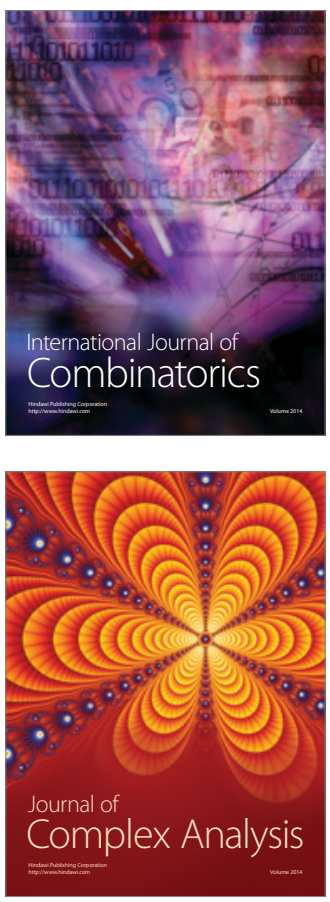

International Journal of

Mathematics and

Mathematical

Sciences
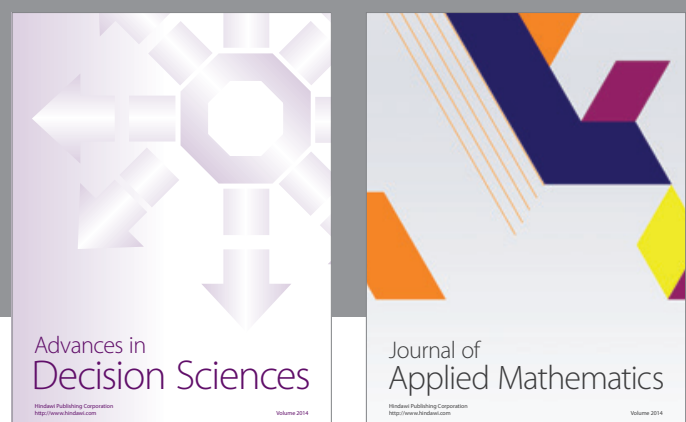

Journal of

Applied Mathematics
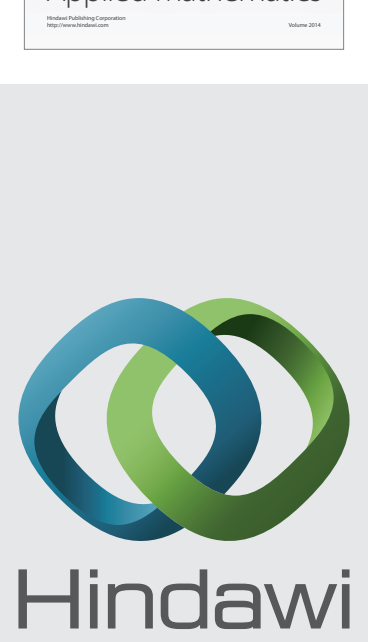

Submit your manuscripts at http://www.hindawi.com
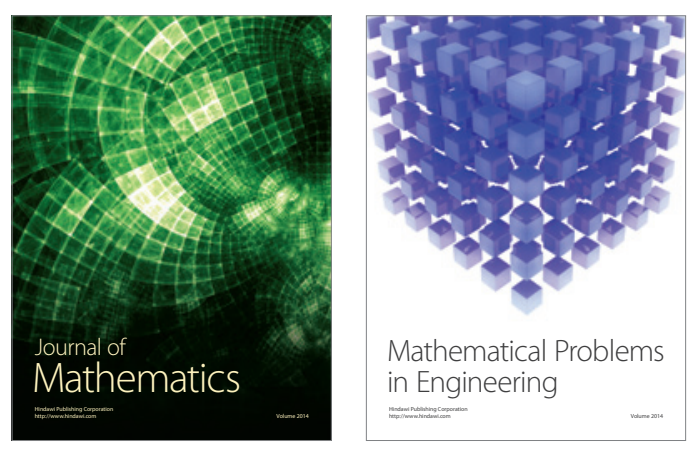

Mathematical Problems in Engineering
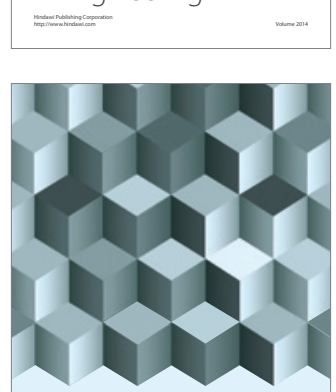

Journal of

Function Spaces
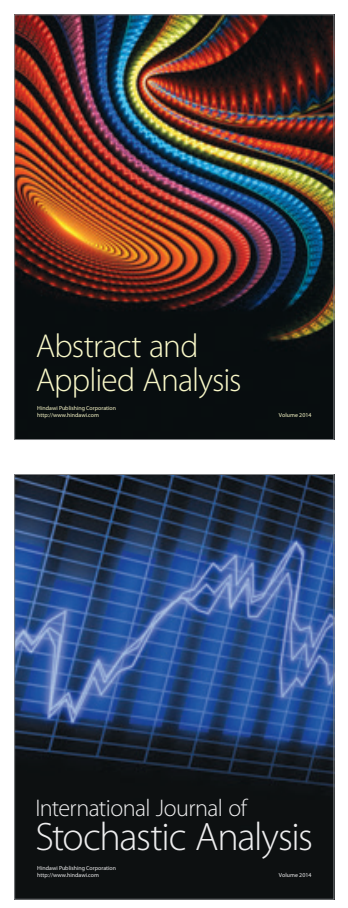

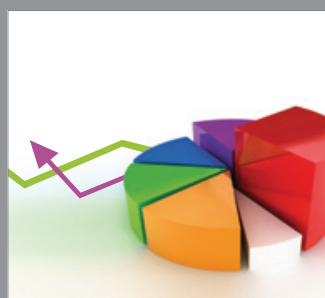

ournal of

Probability and Statistics

Promensencen
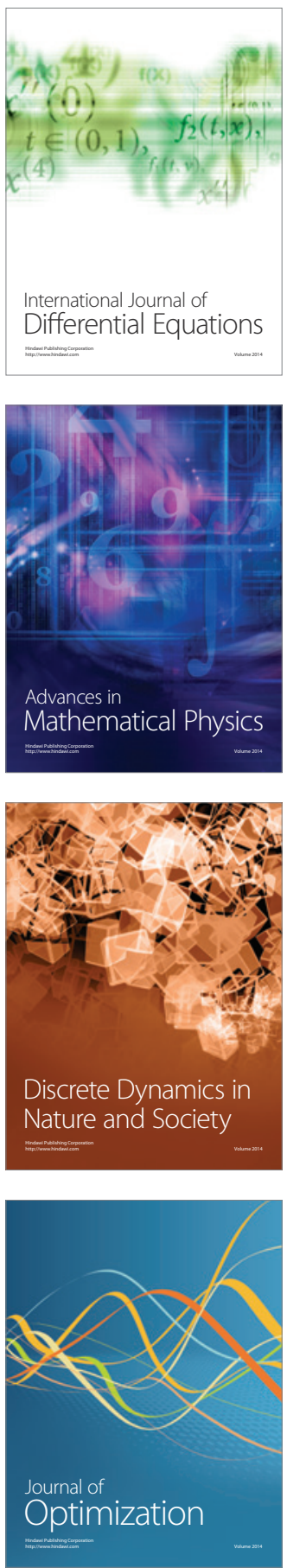